\title{
Treatment of Primary Pleomorphic Soft Tissue Sarcoma of the Extremities
}

\author{
Amir Sternheim ${ }^{*}, 1,2$ Jacob Bickels ${ }^{2}$ and Martin M. Malawer ${ }^{3,4}$ \\ ${ }^{I}$ Investigation performed at the Department of Orthopedic Oncology, Washington Cancer Institute Washington Hospital \\ Center, Washington DC, USA \\ ${ }^{2}$ Department of Orthopedic Oncology, Tel-Aviv Medical Center, Tel-Aviv, Israel \\ ${ }^{3}$ Georgetown University, Washington DC, USA \\ ${ }^{4}$ Pediatric and Surgery Branch, National Cancer Institute, Bethesda, USA
}

\begin{abstract}
Background: Pleomorphic Sarcoma is the most common histologic subtype of high grade soft tissue sarcoma involving the extremities.

Methods: This retrospective study analyzed 135 patients presenting with primary Pleomorphic Sarcomas which were treated at a single referral institute between 1980 and 2006. Data was collected from an institutional database and univariate and multivariate analysis was conducted regarding significant risk factors which influence outcome. 125 (93\%) patients underwent wide limb sparing resection, primary amputation was necessary in $10(7 \%)$ patients. 52 patients $(39 \%)$ received post-operative radiation therapy alone, $23(17 \%)$ received adjuvant chemotherapy alone and 37 patients $(27 \%)$ received both chemotherapy and radiation therapy. 23 patients $(17 \%)$ received no adjuvant therapy.

Results: The 5-year disease specific survival rate was $74 \% \pm 4 \%$, metastatic rate $29 \%$ and local recurrence rate was $22 \% \pm 4 \%$. Significant prognostic factors negatively influencing survival were a local recurrence, large size, deep tumors, close margins and proximal location. Risk factors for a local recurrence were a superficial lesion, upper extremity and extra-compartmental location. Induction chemotherapy did not show a survival or a local recurrence benefit. Chemotherapy induced tumor necrosis rates did not correlate with a survival benefit.

Conclusions: Anatomic location of Pleomorphic soft tissue sarcoma influences local recurrence and survival rates. Induction chemotherapy did not show a survival benefit. Chemotherapy induced tumor necrosis rate does not correlate with survival and therefore should not guide adjuvant treatment decisions.
\end{abstract}

Keywords: Pleomorphic, Soft tissue Sarcoma, Extremities, Prognostic factors, Chemotherapy, Radiation therapy.

\section{INTRODUCTION}

Pleomorphic Sarcoma is the most common type of high grade soft tissue sarcoma of adult life, often presents as an aggressive, large, high grade sarcoma of the extremity [110]. Prognostic risk factors include tumor size, depth and proximal location. Local control of the primary tumor is highly dependent on the surgical margin achieved at the time of surgical resection; which remains the mainstay of treatment. There is less consensus on the role of adjuvant therapy in the form of radiation therapy and chemotherapy due to the minimally significant influence on survival and the potential systemic and local side effects $[11,12]$.

Adjuvant radiation therapy, can improve local tumor control, presumably by extending the surgical margin. Radiation has also been used as an induction treatment to induce tumor shrinkage and facilitate surgical resection [13]. Chemotherapy can have both local and systemic effects. Neo-adjuvant chemotherapy has not shown a clear survival benefit [14], a recent study compared neo-adjuvant

*Address correspondence to this author at the Orthopedic Oncology, Tel-Aviv Medical Center, 660 Briar Hill Ave, Toronto, Canada; Tel: (647)980-1471; Fax: (416)-586-8673; E-mail: Amirsternheim@gmail.com chemotherapy $(\mathrm{n}=71)$ and resection to resection only for high grade sarcomas showed some survival benefit $(p<0.02)$ only for large sarcomas $>10 \mathrm{~cm} \mathrm{[15].}$

We examined our treatment experience with induction chemotherapy, radiation therapy in adjunction to surgical resection in 135 patients who presented with primary Pleomorphic Sarcoma of the extremities. We focused on several key questions:

1. What are the key independent and dependant variables influencing survival and local recurrence?

2. What is the influence of anatomic location from which the Pleomorphic Sarcoma originates?

3. What is the influence of chemotherapy, induction chemotherapy and does chemotherapy induced tumor necrosis rate on survival?

\section{MATERIALS AND METHODS}

Between 1980 and 2006, 135 patients with localized primary Pleomorphic Sarcoma of the extremity underwent complete resection of their disease at a single sarcoma referral center and were followed prospectively. This study is a retrospective database and chart review of that data. 
Inclusion criteria were Pleomorphic Sarcoma arising in the extremities. Exclusion criteria were recurrent sarcomas and tumors of the chest wall and back. Initially 155 patients well located. Fifteen patients were excluded from the study as they presented with recurrent Pleomorphic Sarcoma. Five additional patients were excluded since they presented with tumors of the back and chest wall. 135 patients with primary Pleomorphic Sarcoma of the extremities served as the final study group.

Clinical, pathologic and treatment factors and their impact on survival and local recurrence were analyzed. These included age $(<50$ or $\geq 50)$, gender, primary presenting symptom, histologic grade, tumor size $(\leq 5 \mathrm{~cm}, 5-10 \mathrm{~cm}$, or more than $10 \mathrm{~cm}$ ), tumor depth, anatomic location, resection margins, chemotherapy treatment, chemotherapy induced tumor necrosis rate and radiation therapy.

Tumor location was considered proximal if it was located proximal to the knee or elbow. Compartmental tumors arose within muscle groups whereas extra-compartmental space tumors were located in the popliteal space, femoral triangle, sartorial canal, cubital fossa, axilla and shoulder. Tumors were considered deep if they were invading or deep to the muscle fascia and superficial if they were completely within the subcutaneous tissue. Initial clinical presentation was recorded as either painless mass, painful mass, neurologic symptoms or other findings (deep vein thrombosis, pulmonary embolus). Histologic grade according to the National Cancer Institute guidelines was based on the degree of differentiation, pleomorphism, cellularity, amount of stroma, necrosis, vascularity and the number of mitosis.

Treatment variables analyzed for their influence on prognosis included surgical resection technique; limb sparing surgery and primary amputation. Microscopic margins were defined as positive if they were within $1 \mathrm{~mm}$ from the edge, close if tumor was present between $1 \mathrm{~mm}$ and $1 \mathrm{~cm}$ from the edge of the pathology specimen and negative if they were more than $1 \mathrm{~cm}$ from the resection margin. Post-operative complications were recorded as delayed but spontaneous wound healing and those necessitating wound revisions.

Chemotherapy treatment included a post-operative chemotherapy only protocol, a preoperative (induction) only protocol and a combined pre and postoperative chemotherapy protocol soft tissue sarcoma protocol (STSP) with adriamycin, intra-arterial cisplatin, and ifosfamide. The different chemotherapy protocols are described in appendix 1. Chemotherapy induced tumor necrosis rates were recorded from the resected specimen. The necrosis rate was determined by cutting the specimen in a grid like fashion and examining each mapped out cut. The extent of necrosis was determined relative to the percentage of residual viable tissue on each slide. Quantitative analysis was performed by averaging the values for extent of necrosis and residual tumor. 40 patients who received combined pre and postoperative chemotherapy were divided into 4 groups according to their tumor necrosis rates $(<50 \%, 51 \%-80 \%$, $81 \%-95 \%,>95 \%)$ and analyzed for survival. Pathologic diagnosis of Pleomorphic Sarcoma was based on the initial core needle biopsy and the gross specimen at the time of definitive resection.
Radiation treatment was tailored for every patient and anatomic tumor location but was always given postoperatively, the general dose for superficial lesions and deep lesions was 5500-6500 cGy.

Survival times were calculated from the time of definitive resection. Local recurrence times were calculated at the time of resection of the first recurrence even when the patients were salvaged successfully. Follow-up data were obtained from our prospective database, patient chart and telephone contact with patients.

This study was approved by the appropriate institutional review board. Adjuvant chemotherapy and/or radiation therapy was administered to some patients at the discretion of the multidisciplinary soft tissue sarcoma committee or as part of institutional review board- approved clinical trials.

Comparison between two groups was calculated with Fishers exact test. Survival and survivorship free of local recurrence were estimated as a function of time from surgical resection by using the Kaplan-Meier method and is presented as percentages. Comparisons of survival between groups were calculated by using the log rank tests. Associations of continuous risk factors with survival and the multivariate association of combinations of risk factors with survival were estimated using Cox's proportional hazards model. The relative risk associated with each factor in the model is reported as well as the confidence intervals (CI) of the relative risk. Variables were considered statistically significant at $\mathrm{p}<0.05$. Statistical analyses were performed using SAS/STAT software (SAS Inc., Cary, NC).

\section{RESULTS}

\section{Demographics}

Demographic clinical, pathologic and treatment characteristics for 135 patients with primary Pleomorphic Sarcoma are presented in Table 1. 55\% of patients were male. Median age was 59 years (range: 17-87 years). Mean follow-up time was 64 months. Ninety percent had a minimum follow-up of two years. A majority of tumors were high grade (127 patients; 94\%). Telangiectatic transformation in Pleomorphic Sarcoma; a large central hemorrhagic and necrotic fluid mass with tumor nodules, was documented in 8 patients $(5 \%)$. The anatomic locations of the lesions are shown in Table 2. Tumors were located in the lower extremity in 98 patients $(73 \%)$.

\section{Surgical Resection, Chemotherapy and Radiation Therapy}

All patients had surgical resection of their sarcoma. Limb sparing surgery was obtainable in 125 patients (93\%) while primary amputation was deemed necessary in 10 patients (7\%). Microscopic resection margins were negative in 83 patients $(61 \%)$. Twenty-three patients $(17 \%)$ had surgical resection as stand alone treatment. Chemotherapy in adjunction to surgical resection was used to treat 23 patients (17\%). Radiation therapy after surgical resection was the treatment in 52 patients $(39 \%)$. All three treatment modalities were used together in 37 patients $(27 \%)$.

Chemotherapy was administered to 60 patients (44\%). Chemotherapy induced tumor necrosis rates were available 
Table 1. Clinical and Pathologic Characteristics in 135 Patients with Primary Pleomorphic Sarcoma of the Extremity

\begin{tabular}{|c|c|c|c|}
\hline Factor & & Number & Percent \\
\hline \multirow[t]{2}{*}{ Gender } & Male & 74 & $55 \%$ \\
\hline & Female & 61 & $45 \%$ \\
\hline \multirow[t]{2}{*}{ Grade } & Low & 8 & $6 \%$ \\
\hline & High & 127 & $94 \%$ \\
\hline \multirow[t]{2}{*}{ Extremity location } & Upper extremity & 37 & $27 \%$ \\
\hline & Lower extremity & 98 & $73 \%$ \\
\hline \multirow[t]{3}{*}{ Size category } & less than $5 \mathrm{~cm}$ & 49 & $36 \%$ \\
\hline & $5-10 \mathrm{~cm}$ & 48 & $36 \%$ \\
\hline & more than $10 \mathrm{~cm}$ & 38 & $28 \%$ \\
\hline \multirow[t]{2}{*}{ Depth of tumor } & Deep & 97 & $72 \%$ \\
\hline & Superficial & 38 & $28 \%$ \\
\hline \multirow[t]{2}{*}{$\begin{array}{l}\text { Compartments } \\
\text { (Deep tumors only) }\end{array}$} & Intra-compartmental & 37 & $38 \%$ \\
\hline & Extra-compartmental & 60 & $62 \%$ \\
\hline \multirow[t]{4}{*}{ Initial clinical presentation } & Painful & 20 & $15 \%$ \\
\hline & Non-painful & 109 & $80 \%$ \\
\hline & Neurology & 1 & $1 \%$ \\
\hline & Other symptom & 5 & $4 \%$ \\
\hline \multirow[t]{2}{*}{ Primary amputation rate } & Amputation & 10 & $7 \%$ \\
\hline & Limb sparing surgery & 125 & $93 \%$ \\
\hline \multirow[t]{3}{*}{ Complications } & None & 113 & $84 \%$ \\
\hline & Delayed wound healing & 8 & $6 \%$ \\
\hline & Wound revision & 14 & $10 \%$ \\
\hline \multirow[t]{3}{*}{ Margins } & Negative & 83 & $61 \%$ \\
\hline & Close & 47 & $35 \%$ \\
\hline & Positive & 5 & $4 \%$ \\
\hline \multirow[t]{4}{*}{ Chemotherapy } & Adjuvant & 17 & $13 \%$ \\
\hline & Neoadjuvant & 3 & $2 \%$ \\
\hline & Combined pre and post op. & 40 & $29 \%$ \\
\hline & None & 75 & $56 \%$ \\
\hline \multirow[t]{2}{*}{ Radiation therapy } & Radiation & 89 & $66 \%$ \\
\hline & No & 46 & $34 \%$ \\
\hline
\end{tabular}

for all 40 patients who received the soft tissue sarcoma protocol (STSP) which included both neoadjuvant and adjuvant chemotherapy. Adjuvant radiation therapy was administered post-operatively to a total of 89 patients $(66 \%)$ with external beam therapy.

\section{Postoperative Wound Complications}

Postoperative wound complications occurred in 22 patients $(16 \%)$. Chemotherapy induced minor complications associated with the use of intra-arterial cisplatin included four patients $(2.9 \%)$ who developed myocutaneous necrosis. One other patient developed an arterial thrombosis requiring an embolectomy. All complications resolved and had no impact on surgical procedures performed, wound healing, or ultimate outcome. Minor complications of systemic chemotherapy included manageable neutropenia, thrombocytopenia, anemia, nausea, mucositis, alopecia, weight loss, and minor peripheral neuropathy.

Table 2. Exact Anatomic Location of 135 Extremity Pleomorphic Sarcomas

\begin{tabular}{|c|c|c|c|c|}
\hline & $\mathbf{n}$ & Percent & LR & Deceased \\
\hline Superficial lesions & 38 & 28 & 8 & 3 \\
\hline Thigh anterior compartment & 31 & 23 & 2 & 12 \\
\hline Thigh posterior compartment & 8 & 6 & 1 & 2 \\
\hline Thigh adductor compartment & 6 & 4 & 1 & 3 \\
\hline Popliteal space & 8 & 5 & 1 & 2 \\
\hline Buttock & 5 & 4 & 1 & 2 \\
\hline Femoral triangle space & 5 & 3 & 3 & 3 \\
\hline Leg posterior compartment & 7 & 5 & 2 & 2 \\
\hline Foot & 4 & 3 & & 2 \\
\hline Shoulder & 14 & 11 & 6 & 5 \\
\hline Arm posterior compartment & 3 & 1 & 1 & 1 \\
\hline Forearm & 1 & & & \\
\hline Elbow cubital space & 1 & & & \\
\hline Hand & 1 & & & \\
\hline \multirow[t]{2}{*}{ Other } & 3 & 2 & 1 & \\
\hline & 135 & & 27 & 37 \\
\hline \multicolumn{5}{|l|}{ Anatomic Region } \\
\hline Proximal lower extremity & 77 & & & \\
\hline Proximal upper extremity & 22 & & & \\
\hline Distal lower extremity & 21 & & & \\
\hline \multirow[t]{2}{*}{ Distal upper extremity } & 15 & & & \\
\hline & 135 & & & \\
\hline LR- Local recurrence & & & & \\
\hline
\end{tabular}

\section{Survival Rates and Prognostic Factors}

The overall survival rate was $72 \%$ as 37 patients died of their disease. The 5-year survival rate was $74 \% \pm 4 \%$ and the 10 -year survival was $66 \% \pm 5 \%$. A local recurrence, large tumor size, deep tumors, close margins and proximal location in the extremity were found to have a significant negative prognostic influence on survival on univariate analysis (Table 3). On multivariate analysis local recurrence, 
Table 3. Factors Associated with Local Recurrence Free Survival and Disease Specific Survival

\begin{tabular}{|c|c|c|c|c|c|c|c|c|}
\hline \multirow[b]{2}{*}{ Factor } & \multicolumn{4}{|c|}{ Local Recurrence Free Survival } & \multicolumn{4}{|c|}{ Disease specific survival } \\
\hline & Univariate & Cox Model & Relative Risk & $95 \% \mathrm{CI}$ & Univariate & Cox Model & Relative Risk & $95 \% \mathrm{CI}$ \\
\hline Age $>50$ years & NS & & & & NS & & & \\
\hline Local recurrence & $\mathrm{x}$ & $\mathrm{x}$ & $\mathrm{x}$ & $\mathrm{x}$ & 0.0097 & 0.036 & 2.14 & $1.05-4.37$ \\
\hline Size $>5 \mathrm{~cm}$ & NS & & & & 0.0019 & & & \\
\hline Close Margins & NS & & & & 0.05 & & & \\
\hline High grade & NS & & & & NS & & & \\
\hline Deep location & NS & 0.01 (superficial) & 4.45 & $1.4-14.6$ & 0.0051 & 0.016 & 4.6 & $1.34-15.9$ \\
\hline Chemotherapy treatment & NS & & & & NS & 0.0062 & 0.45 & $0.22-0.96$ \\
\hline Tumor necrosis $>95 \%$ & & & & & NS & & & \\
\hline Upper extremity & 0.0075 & & & & NS & & & \\
\hline Proximal location & NS & & & & 0.033 & & & \\
\hline Extra-compartmental space & 0.0088 & & & & NS & & & \\
\hline NS - Non-significant & & & & & & & & \\
\hline
\end{tabular}

tumor depth and chemotherapy treatment had a significant influence on survival.

Local recurrence occurred in 27 patients who initially had primary resection at our institute. The 5-year survival rate of those 27 was $60 \% \pm 10 \%$ (14 deceased in total) compared to the survival rate of those patients who did not have a local recurrence, 108 patients with a 5-year survival rate of $78 \% \pm 4 \%$ ( 25 deceased in total). Local recurrence was a significant negative predictive factor for survival $(\mathrm{p}<$ 0.0097).

Tumor size was a significant prognostic factor $(\mathrm{p}<$ $0.0019)$, larger tumors faired worse. The 5-year survival rate for tumors smaller than $5 \mathrm{~cm}$ was $88 \% \pm 5 \%$, between 5 and $10 \mathrm{~cm} 71 \% \pm 7 \%$ and those greater than $10 \mathrm{~cm}$ was $60 \% \pm 8 \%$.

Proximal tumor location was significant negative prognostic factor $(\mathrm{p}<0.03)$. The 5-year survival rate for tumors arising in a proximal location was $68 \% \pm 5 \%$ compared to $84 \% \pm 6 \%$ for tumors arising in a distal location.

Close margins were a negative significant prognostic factor $(p<0.05)$. The 5-year survival rate for tumors resected with close margins $(<1 \mathrm{~cm})$ was $63 \% \pm 7 \%$ compared to $81 \% \pm 5 \%$ for those resected with negative $(>1 \mathrm{~cm})$ margins.

Depth of the lesion was a significant risk factor $(\mathrm{p}<0.0051)$. The 5-year survival rate for superficial lesions was $92 \% \pm 6 \%$ compared to $68 \% \pm 7 \%$ for deep lesions. There were a total of 97 (72\%) deep lesions involving muscles and tissues deep to the fascia. $80 \%$ of these tumors were larger than $5 \mathrm{~cm}$. Microscopic margins were negative in $58(59 \%)$, close in $35(36 \%)$ and positive in $4(5 \%)$ of patients.

\section{Local Recurrence and Risk Factors for Local Recurrence}

The local recurrence rate was $20 \%$ (27 patients). The 5 and 10 year local recurrence rates were $22 \% \pm 4 \%$ and $26 \% \pm 5 \%$ accordingly. Nine (33\%) local recurrences were superficial and $18(66 \%)$ were deep. The local recurrence rate among superficial lesions was $24 \%$ while among deep lesions it was $18.5 \%$. Upper extremity disease and disease located in an extra-compartmental space were found to be significant risk factors for local recurrence on univariate analysis (Table $\mathbf{3}$ ).

Tumors in the upper extremity lesions had significantly more local recurrences $(p<0.0075)$, the 5-year upper extremity local recurrence rate was $40 \% \pm 11 \%$ compared to the lower extremity rate of $16 \% \pm 4 \%$.

Extra-compartmental space tumors had significantly more $(p<0.009)$ local recurrences when compared to compartmental tumors, at 5-years the extra-compartmental local recurrence rate was $41 \% \pm 11 \%$ compared to the lower extremity rate of $15 \% \pm 5 \%$.

\section{Metastatic Disease}

Metastatic disease developed in 39 patients (29\%). The initial sites of metastasis were in the lungs in 37 patients, including a patient with mediastinal lymph node lesion. Additional sites were the liver in two patients and the brain and skin in one each. Metastasis to regional lymph nodes was detected in two patients.

\section{Pre and Post-Operative Chemotherapy and its Impact on Tumor Necrosis Rate and Survival}

Chemotherapy induced tumor necrosis and its' impact on survival (Table 4) was analyzed in 40 patients who were treated according to our soft tissue sarcoma chemotherapy protocol (STSP) and received chemotherapy both before and after resection. Log-Rank comparison of survival analysis was also found to be non-significant for both a $90 \%$ tumor necrosis rate cut-off $(\mathrm{P}-0.093)$ and a $95 \%$ cut-off $(\mathrm{P}-0.081)$.

Combined induction and adjuvant chemotherapy was compared to no chemotherapy by analyzing two groups of 40 patients each, adjusted for deep lesions, large $(>5 \mathrm{~cm})$, high grade Pleomorphic Sarcoma. There was no significant difference in survival between the two groups (p- 0.1 ), the 5year survival was $75 \% \pm 7 \%$ with chemotherapy compared to 
$61 \% \pm 9 \%$ without chemotherapy. There was also no significant difference in local recurrence rates between the two groups (p- 0.143).

Table 4. No Statistically Significant Relationship Between Rate of Tumor Necrosis and Survival in 40 Patients Receiving Pre and Post Operative Chemotherapy

\begin{tabular}{|ccc|c|}
\hline \% Tumor Necrosis & Alive & Dead & Total \\
\hline \hline $50 \%$ and less & 8 & 5 & 13 \\
$51 \%-80 \%$ & 5 & 1 & 6 \\
$81 \%-95 \%$ & 6 & 2 & 8 \\
More than $95 \%$ & 11 & 2 & 13 \\
\hline & 30 & 10 & 40 \\
\hline
\end{tabular}

\section{Impact of Resection Margins and Radiation Therapy on Survival}

Patients were divided into four groups and analyzed for the influence of surgical margin status (close vs negative) with or without radiation therapy on survival: Group 1radiation treatment and negative margins (47 patients), Group 2- no radiation treatment and negative margins (36 patients), Group 3- radiation treatment and close margins (42 patients), Group 4- no radiation treatment and close margins (10 patients). There was no significant difference (P-0.21) in survival rates between the four groups. There was also no significant difference $(\mathrm{P}-0.74)$ in the survival rates of only those patients who had local recurrence between the four groups.

\section{DISCUSSION}

The current study joins several previous studies of extremity Pleomorphic Sarcoma (Table 5) [13, 16-18]. Although these studies span several decades, their outcomes are similar. The 5-year survival was $74 \%$, the distant recurrence rate was $29 \%$ and the local recurrence rate was $20 \%$. Risk factors negatively influencing survival were a local recurrence, large tumor size, tumor depth and close microscopic resection margins. Risk factors for a local recurrence were superficial lesions, upper extremity location and an extra-compartmental location. Margins status did not correlate with local recurrence.

A significant weakness of this study is that patients did not receive uniform treatment. The treatment algorithm for high grade soft tissue sarcomas at our institution over the last two decades has been wide resection and post-operative radiation therapy. Chemotherapy was initially limited to patients with high grade STS of the extremities and pelvis judged to be unresectable with wide margins because of anatomic location, size and proximity to blood vessels. These indications were later expanded to include all patients with extremity high grade soft tissue sarcoma that were deemed medically fit for chemotherapy by a multidisciplinary team. Initially reported in osteosarcoma and later in STS, induction chemotherapy may permit limb salvage by inducing tumor shrinkage. A group of 40 patients with large deep lesions in healthy young patients were treated with wide resection and combined pre and postoperative chemotherapy. Elderly or unhealthy patients were treated with wide resection and radiation therapy thus avoiding the short term possible side effects of chemotherapy (52 patients). Adjuvant therapy was at times completely discarded at the discretion of the multidisciplinary soft tissue sarcoma committee based on small tumor size, negative margins of resection and superficial location that affords early diagnosis of any local recurrence (23 patients).

Local recurrence was shown to be a significant risk factor for survival on both uni- and multi-variate analysis. Patients who did not have a local recurrence had a 5-year survival rate of $78 \%$ compared to those who did have a local recurrence where survival dropped to $60 \%$. This has previously been reported by Salo et al. but unique to this study was the fact that these were patients who initially presented with primary disease and not patients who presented with a local recurrence after prior resection at a different institution, thus giving stronger validity [16]. The question remains, can we avoid a local recurrence by better surgical technique (negative margins) or with additional treatment such as radiation therapy. We found we could not influence local recurrence or survival (P-value 0.74) of patients with wider surgical margins or additional radiation therapy. Weiss et al. have previously shown that amputation in Pleomorphic Sarcoma avoids the local recurrence but not the distal recurrence or survival, when compared to limb sparing surgery [2].

The primary anatomic location of the lesion influences the risk of local recurrence and survival of Pleomorphic Sarcoma. The quadriceps muscle is by far the most common location (32\%) of all deep lesions, this was previously reported by Pritchard et al. [17]. Local recurrence in the quadriceps is relatively rare (7\% of all local recurrences) but mortality is very high ( $32 \%$ of all deaths). Tumors of the shoulder on the other hand were $11 \%$ of all tumors, yet they

Table 5. Literature Review of Four Studies Focused on Pleomorphic Sarcoma Outcome Rates and Prognostic Factors Found to Influence Survival

\begin{tabular}{|c|c|c|c|c|c|c|c|c|c|c|}
\hline Study & No. of Patients & Local Recurrence (\%) & Metastasis (\%) & 5 Year Survival & Age & Local Recurrence & Size & Margins & Grade & Depth \\
\hline Salo et al. 1999 & 239 & 19 & 35 & 65 & No & Yes & Yes & & Yes & Yes \\
\hline Le Doussal et al. 1996 & 216 & 37 & 36 & 70 & Yes & & Yes & Yes & & Yes \\
\hline Zagars et al. 1996 & 271 & 21 & 31 & 68 & & & Yes & & & \\
\hline Pritchard et al. 1993 & 220 & 32 & 42 & 57 & Yes & No & Yes & Yes & No & Yes \\
\hline Current study & 135 & 20 & 29 & 74 & No & Yes & Yes & Yes & No & Yes \\
\hline
\end{tabular}


were double that rate in local recurrences $(22 \%)$, but mortality on the other hand was only $14 \%$ of all deaths.

Pleomorphic Sarcoma arising in extra-compartmental spaces (popliteal space, femoral triangle and shoulder) did not have a lower survival rate when compared to compartmental tumors. Similar findings have been previously reported by Eilber et al. [19]. Proximal tumors did have a significantly worse prognosis when compared to distal lesions ( $\mathrm{p}$-value 0.03).

The role of chemotherapy in high grade soft tissue sarcoma remains unclear, a recent meta-analysis confirmed marginal efficacy with respect to recurrence and survival [11]. There was no significant survival or local recurrence rate advantage to chemotherapy treatment when compared to a group that was not treated with chemotherapy, adjusted for high grade, deep, large, primary Pleomorphic Sarcoma. Chemotherapy treatment was a significant protective factor for survival on multi-variate analysis but not on uni-variate analysis. A possible explanation is that uni-variate analysis may be biased by other differences in risk factors not accounted for in the comparison. Survival for the chemotherapy group may have been even grimmer without the chemotherapy. Chemotherapy in soft tissue sarcoma remains a controversial issue in extremity soft tissue sarcoma after more than twenty years as it has shown borderline efficacy at best [15].

We continue to use induction chemotherapy to decrease the size of the tumor thus turning a previously unresectable tumor that necessitated amputation into a resectable tumor amenable for limb sparing surgery. In initially resectable tumors, this tumor shrinkage effect minimizes the soft tissue resection needed for limb sparing resection thus improving the functional outcome. In addition, chemotherapy through fibrosis solidifies the tumors pseudocapsule thereby simplifying the resection. This was previously suggested by Pritchard et al. by using preoperative radiation instead of chemotherapy [17]. We prefer induction chemotherapy to radiation therapy because the latter is associated with higher wound complication rates [12].

Chemotherapy induced tumor necrosis has been used as a tool to evaluate the response of the tumor to treatment midway through treatment. The current study found no significant correlation between the rate of tumor necrosis and survival. This variable is a by product of induction chemotherapy. Tumor necrosis rates of $90 \%$ and $95 \%$ and higher have been considered a good response to therapy. Eilber et al. have shown correlation between chemotherapy induced tumor necrosis rates above $95 \%$ and better survival rates in soft tissue sarcoma [20]. Our outcomes join a growing body of evidence including Menendez et al. who recently published their similar results that do not show correlation between necrosis rates and survival [21].

External beam radiation therapy was used to treat 89 (66\%) patients postoperatively. Preoperative radiation therapy has higher wound complication rates $(35 \%)$ compared to post-operative treatment (17\%) [12]. Preoperative radiation aids in decreasing tumor size and facilitating surgical resection. Adjuvant radiation therapy, can improve local tumor control, presumably by extending the surgical margin. We could not show a significant difference in local recurrence rate $(\mathrm{p}-0.21)$ between patients who received radiation therapy and had negative resection margins and a second group who did not have radiation therapy and had close resection margins. In a randomized prospective study, O'Sullivan et al, showed improved survival with preoperative radiation therapy when compared to post-operative radiation but this came with a higher rate of wound complications [12].

Deep tumors are known to have a significant negative prognostic influence on survival [13, 16-18]. Evidently, the overall survival rates between studies as shown in Table 5 are greatly influenced by the relative percentage of superficial ( $>90 \%$ survival) and deep lesions (65\% survival). One quarter of Pleomorphic Sarcomas have been reported to present as superficial lesions involving the sub-cutis and fascia, the rate in this study was $30 \%$, which had impact on the favorable general survival rates $[2,16]$.

It is difficult to differentiate between the influence of size and depth on outcome. Deep sarcomas accounted for more than two thirds of all lesions in this study. They were larger in size, with $80 \%$ greater than $5 \mathrm{~cm}$, this is in complete contrast to superficial lesions $(80 \%<5 \mathrm{~cm})$. In univariate analysis both deep lesions and large lesions were significant risk factors ( $p$-value 0.005 and 0.0029 accordingly), yet in multivariate analysis only deep tumors were a significant risk factor ( $\mathrm{p}$-value 0.0157 ) but not size. 5-year survival rates for patients who had a local recurrence were $61 \%$ similar to the $65 \%$ survival for all deep lesions. This is in complete contrast to superficial lesions where local recurrence is followed by a lower survival rate. This group of deep, often large tumors benefit from induction chemotherapy as this simplifies limb sparing resection and according to Grobmyer et al. influences survival particularly in large tumors greater than $10 \mathrm{~cm}[15]$.

Interestingly, 5\% of Pleomorphic Sarcomas undergo such extensive spontaneous hemorrhage and central necrosis that they present clinically as a fluctuant mass, this finding was initially reported by Weiss et al. almost 30 years ago [2]. We found a similar rate of $5 \%$ of these lesions which we term 'Telangiectatic' transformation in soft tissue sarcomas. They are a distinct variant of aggressive high grade sarcoma [22].

\section{CONCLUSIONS}

The following treatment recommendations represent our practical interpretation of the study results.

1. The role of chemotherapy remains unclear in Pleomorphic Sarcomas. Induction chemotherapy may not influence survival but may enable limb sparing surgery in some tumors initially deemed unresectable; this is a major role change for induction chemotherapy. In all other cases primary resection of the tumor should precede all other treatment modalities.

2. Chemotherapy induced tumor necrosis rate does not correlate with survival and should therefore not influence adjuvant treatment decisions.

3. Tumor depth is a risk factor in Pleomorphic Sarcoma for both survival and local recurrence. 
4. Anatomic location influences survival and local recurrence.

\section{ACKNOWLEDGEMENT}

None declared.

\section{CONFLICT OF INTEREST}

None declared.

\section{REFERENCES}

[1] Enzinger FM. Malignant fibrous histiocytoma 20 years after Stout. Am J Surg Pathol 1986; 10(Suppl 1): 43-53.

[2] Weiss SW, Enzinger FM. Malignant fibrous histiocytoma: an analysis of 200 cases. Cancer 1978; 41: 2250-66.

[3] Ozzello L, Stout AP, Murray MR. Cultural characteristics of malignant histiocytomas and fibrous xanthomas. Cancer 1963; 16: $331-44$.

[4] O'Brien JE, Stout AP. Malignant Fibrous Xanthomas. Cancer 1964; 17: 1445-55.

[5] Randall RL, Albritton KH, Ferney BJ, Layfield L. Malignant fibrous histiocytoma of soft tissue: an abandoned diagnosis. Am J Orthop 2004; 33: 602-8.

[6] Binder SW, Said JW, Shintaku IP, Pinkus GS. A histiocyte-specific marker in the diagnosis of malignant fibrous histiocytoma. Use of monoclonal antibody KP-1 (CD68). Am J Clin Pathol 1992; 97: $759-63$.

[7] Burgdorf WH, Duray P, Rosai J. Immunohistochemical identification of lysozyme in cutaneous lesions of alleged histiocytic nature. Am J Clin Pathol 1981; 75: 162-7.

[8] Choong PF, Mandahl N, Mertens F, et al. 19p+ marker chromosome correlates with relapse in malignant fibrous histiocytoma. Genes Chromosomes Cancer 1996; 16: 88-93.

[9] Churg AM, Kahn LB. Myofibroblasts and related cells in malignant fibrous and fibrohistiocytic tumors. Hum Pathol 1977; 8: 205-18.

[10] Dahlin DC, Unni KK, Matsuno T. Malignant (fibrous) histiocytoma of bone--fact or fancy? Cancer 1977; 39: 1508-16.
[11] Pervaiz N, Colterjohn N, Farrokhyar F, et al. A systematic metaanalysis of randomized controlled trials of adjuvant chemotherapy for localized resectable soft-tissue sarcoma. Cancer 2008; 113: 573-81.

[12] O'Sullivan B, Davis AM, Turcotte R et al. Preoperative versus postoperative radiotherapy in soft-tissue sarcoma of the limbs: a randomised trial. Lancet 2002; 359: 2235-41.

[13] Zagars GK, Mullen JR, Pollack A. Malignant fibrous histiocytoma: outcome and prognostic factors following conservation surgery and radiotherapy. Int J Radiat Oncol Biol Phys 1996; 34: 983-94.

[14] Henshaw RM, Priebat DA, Perry DJ, et al. Survival after induction chemotherapy and surgical resection for high-grade soft tissue sarcoma. Is radiation necessary? Ann Surg Oncol 2001; 8: 484-95.

[15] Grobmyer SR, Maki RG, Demetri GD et al. Neo-adjuvant chemotherapy for primary high-grade extremity soft tissue sarcoma. Ann Oncol 2004; 15: 1667-72.

[16] Salo JC, Lewis JJ, Woodruff JM, et al. Malignant fibrous histiocytoma of the extremity. Cancer 1999; 85: 1765-72.

[17] Pritchard DJ, Reiman HM, Turcotte RE, Ilstrup DM. Malignant fibrous histiocytoma of the soft tissues of the trunk and extremities. Clin Orthop Relat Res 1993; 58-65.

[18] Le Doussal V, Coindre JM, Leroux A, et al. Prognostic factors for patients with localized primary malignant fibrous histiocytoma: a multicenter study of 216 patients with multivariate analysis. Cancer 1996; 77: 1823-30.

[19] Eilber FC, Eckardt JJ, Rosen G et al. Large, deep, high-grade extremity sarcomas: treating tumors of the flexor fossae. Surg Oncol 1999; 8: 211-4

[20] Eilber FC, Rosen G, Eckardt J, et al. Treatment-induced pathologic necrosis: a predictor of local recurrence and survival in patients receiving neoadjuvant therapy for high-grade extremity soft tissue sarcomas. J Clin Oncol 2001; 19: 3203-9.

[21] Menendez LR, Ahlmann ER, Savage K, et al. Tumor necrosis has no prognostic value in neoadjuvant chemotherapy for soft tissue sarcoma. Clin Orthop Relat Res 2007; 455: 219-24.

[22] Sternheim A, Jin X, Shmookler B et al. 'Telangiectatic' transformation in soft tissue sarcomas. a clinicopathology analysis of an aggressive feature of high-grade sarcomas. Ann Surg Oncol 2008; 15: $345-54$. 